\title{
Arthroscopic patterns of the poster-medial aspect of the knee joint: classification of the gastrocnemius- semimembranosus gateway and its relationship with Baker's cyst
}

\author{
Vittorio Calvisi ${ }^{1}$ \\ Carmine Zoccali ${ }^{2}$ \\ 1 Department of Orthopaedic and Traumatology \\ School, University of L'Aquila, Italy \\ 2 Regina Elena National Cancer Institute, \\ Oncological Orthopedic Department, Rome, Italy \\ Corresponding author: \\ Vittorio Calvisi \\ Department of Orthopaedic and Traumatology \\ School, University of L'Aquila \\ Piazzale Salvatore Tommasi 1 \\ 67100 L'Aquila, Italy \\ E-mail: vittorio.calvisi@cc.univaq.it
}

\section{Summary}

Background: The gastrocnemius-semimembranosus bursa may communicate with the knee joint. The arthroscopic anatomy of the posteromedial aspect varies depending on the angle of the oblique popliteal ligament, the level at which it crosses the medial gastrocnemius tendon, and its relationship with the capsular joint and synovia.

The aim of this paper is to identify possible patterns, and to evaluate their characteristics and their relationship with Baker's cyst.

Methods: data archived from 185 consecutive arthroscopies were evaluated; an anatomic description and classification was carried out; the percentages of association with $\mathrm{BC}$ and the associated pathologies were reported.

Results: The different anatomies were classified into six groups based on the relationship above the medial gastrocnemius tendon, the capsular joint and synovia. The prevalence of Baker's cyst was $\mathbf{2 8 . 3} \%$. The main associated intra-articular pathological condition was the contemporary presence of a meniscal tear and chondropathy. Conclusion: Exploration of the posterior aspect of the knee must be performed routinely. Knowing the possible anatomy patterns of the posteromedial arthroscopic aspect of the knee joint could help to identify the cyst and its gateway, thus facilitating its treatment.

Level of the evidence: III.

KEY WORDS: arthroscopy, arthroscopic portal, Baker's cyst, knee arthroscopic anatomy.

\section{Introduction}

In the popliteal fossa, six bursae can be identified ${ }^{1}$. Even though all six can undergo inflammation, the Baker's cyst $(\mathrm{BC})$ is the most frequently involved in the inflammatory process ${ }^{2,3}$.

It was described in 1877 by Baker as a distention of the popliteal bursa 4 .

The BC is located in a specific area between the Oblique Popliteal Ligament (OPL) reinforced by an expansion of the semimembranosus tendon and the medial tendon of the gastrocnemius ${ }^{5}$.

When such a communication exists, knee joint effusions may replete the gastrocnemius-semimembranosus bursa (GSB) with synovial fluid and if the fluid outflow is hindered by an unidirectional valve-like mechanism, the GSB enlarges, giving rise to a pseudocystic cavity referred to as Baker's cyst ${ }^{1}$.

The arthroscopic anatomy varies depending on the angle of the OPL, the level at which it crosses the medial gastrocnemius tendon, and its relationship with the capsular joint and synovia.

The aim of this paper is to describe the arthroscopic anatomy of the posterior aspect of the knee joint, and to perform a classification of the possible patterns and evaluate their relationship with the presence of the $\mathrm{BC}$, in order to facilitate its recognition and the all-inside suture technique for suturing its gateway ${ }^{6}$.

\section{Materials and methods}

All arthroscopies performed at a university research hospital between January 2013 and December 2014 were reviewed. The presenting cohort comprised 201 patients - 142 males $(76.6 \%)$ and 59 females $(23.4 \%)$ - with an average age of 39.9 years (range 13-80, SD 15.51).

Every arthroscopy was performed with a $30^{\circ}$ lens and a $70^{\circ}$ lens to enable the visualization of the posteriormedial part of the knee. A high anterolateral portal was established and a diagnostic arthroscopy of the knee joint was carried out; then the arthroscope was inserted between the medial condyle and the posterior cruciate ligament through the intercondylar notch with the knee flexed at $90^{\circ}$; this portal is particularly advantageous for the visualization of the gateway to the GSB because the orifice opens cranially.

The 16G trial needle was inserted into the posteromedial compartment to explore the anatomy and palpate the gateway to the GSB. The posteromedial por- 
tal was subsequently identified by direct visualization and with the aid of a trial needle. In 16 cases this technique was not possible because of the presence of osteophytes, so the patients were excluded. For the remaining 185 knees, an anatomic description of the posterior aspect of the knee joint was made, and the percentages of association with $\mathrm{BC}$ and the associated pathologies were reported.

The different anatomical patterns were classified into six groups.

\section{Statistical analysis}

The $x^{2}$-test was used to evaluate the association between the anatomical aspect and the presence of BC (SAS/STAT ${ }^{\circledR}$ software was used for this purpose).
The procedures were in accordance with the ethical standards of the responsible committee on human experimentation and with the Helsinki Declaration of 1975 , as revised in 2000 . No funding was received for publication of the paper. The Authors declare no conflict of interest ${ }^{7}$.

\section{Results}

Six types of possible patterns were identified (Fig. 1). Their characteristics and relative frequencies are reported in Table I.

In 57 out of 185 cases (28.3\%) the BC was present (average age 44.1 years, range 13-75). The average ages of the different subgroups are reported in Figure 2 . The lesions associated with the $\mathrm{BC}$ are shown in Figure 3.
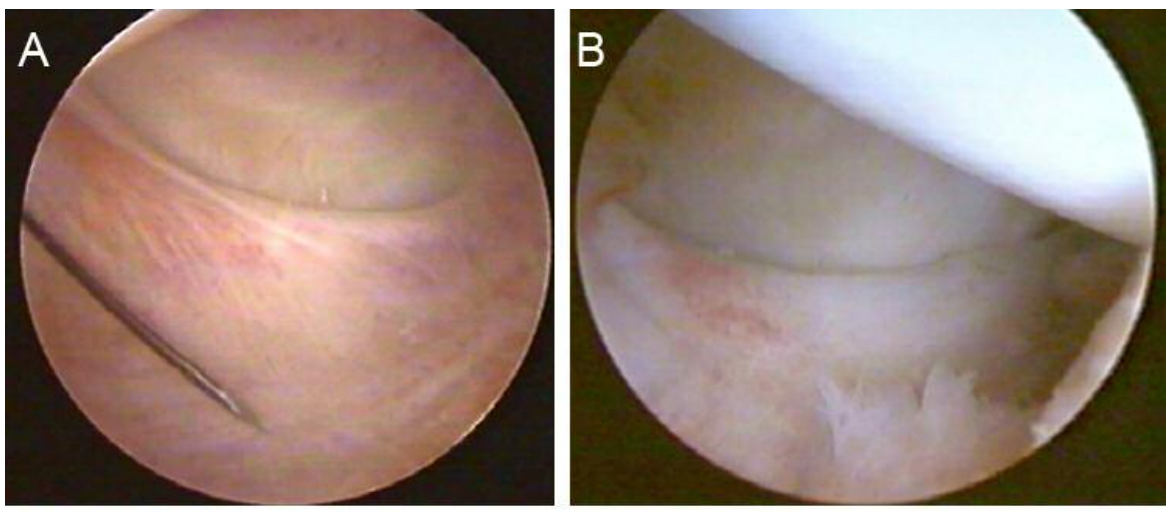

Figure 1. The six patterns described: A: the classic form (high shape); B: the horizontal form; C: plica with small communicating hole; $\mathrm{D}$ : veil form; E: simple plica; F: wide type.
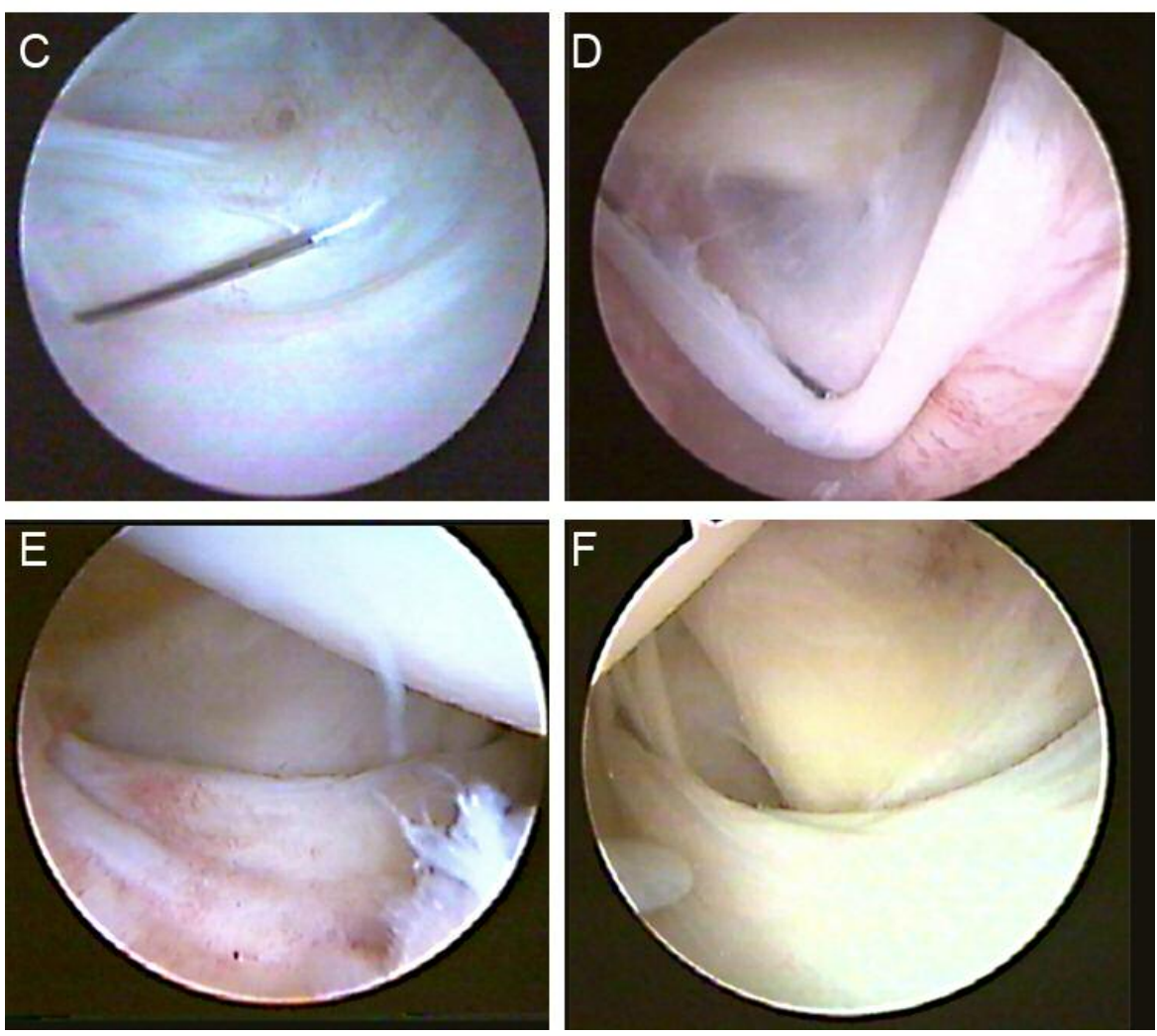
Table I. The arthroscopic patterns of the postero-medial aspect of the knee joint.

\begin{tabular}{|c|c|c|c|}
\hline & Shape & Frequency & Anatomic description \\
\hline Type A & $\begin{array}{l}\text { High shape } \\
\text { or Classic } \\
\text { form (Fig.1A) }\end{array}$ & $16 \%$ & $\begin{array}{l}\text { The most common form. The semimembranosus recurrent tendon crosses the } \\
\text { medial gastrocnemius tendon very proximally. The popliteal cyst is present } \\
\text { in } 31 \% \text { of cases. }\end{array}$ \\
\hline Type B & $\begin{array}{l}\text { Horizontal } \\
\text { (Fig.1B) }\end{array}$ & $8,8 \%$ & $\begin{array}{l}\text { The semimembranosus recurrent tendon runs transversally or in an arc. } \\
\text { A communicating popliteal cyst is present in } 33.4 \% \text { of cases. }\end{array}$ \\
\hline Type C & $\begin{array}{l}\text { Plica with a } \\
\text { small commu- } \\
\text { nicating hole } \\
\text { (Fig.1C) }\end{array}$ & $6.0 \%$ & $\begin{array}{l}\text { The popliteal cyst is always present and it communicates with the joint through a } \\
\text { small hole (in } 0.55 \% \text { of cases the communication was through two holes). }\end{array}$ \\
\hline Type D & $\begin{array}{l}\text { Veil type } \\
\text { (Fig.1D) }\end{array}$ & $4,4 \%$ & $\begin{array}{l}\text { The popliteal cyst is always present; a veil is present in the communicating door; } \\
\text { the posterior anatomic structures can be seen in transparency in the background. } \\
\text { In } 75 \% \text { of cases, the veil is complete; in } 25 \% \text { of cases, it is incomplete. }\end{array}$ \\
\hline Type E & $\begin{array}{l}\text { Simple Plica } \\
\text { (s) (Fig.1E) }\end{array}$ & $3,9 \%$ & $\begin{array}{l}\text { Single plica with pouch }(1.1 \%) \text { : an extroflexed capsular pouch is present under } \\
\text { the semimembranosus recurrent tendon. The BC is never present. Double plica } \\
(2.2 \%) \text { : Proximally to the semimembranosus recurrent tendon there are two plicae } \\
\text { which seem like a cystic hiatus but the cyst was never present. Triple plica } \\
(0.55 \%) \text { : Similar to the previous form but with three capsular plicae. }\end{array}$ \\
\hline Type F & $\begin{array}{l}\text { Wide type } \\
\text { (Fig.1F) }\end{array}$ & $1,1 \%$ & $\begin{array}{l}\text { The popliteal cyst is always present and it communicates with the joint through } \\
\text { a large gap. }\end{array}$ \\
\hline
\end{tabular}

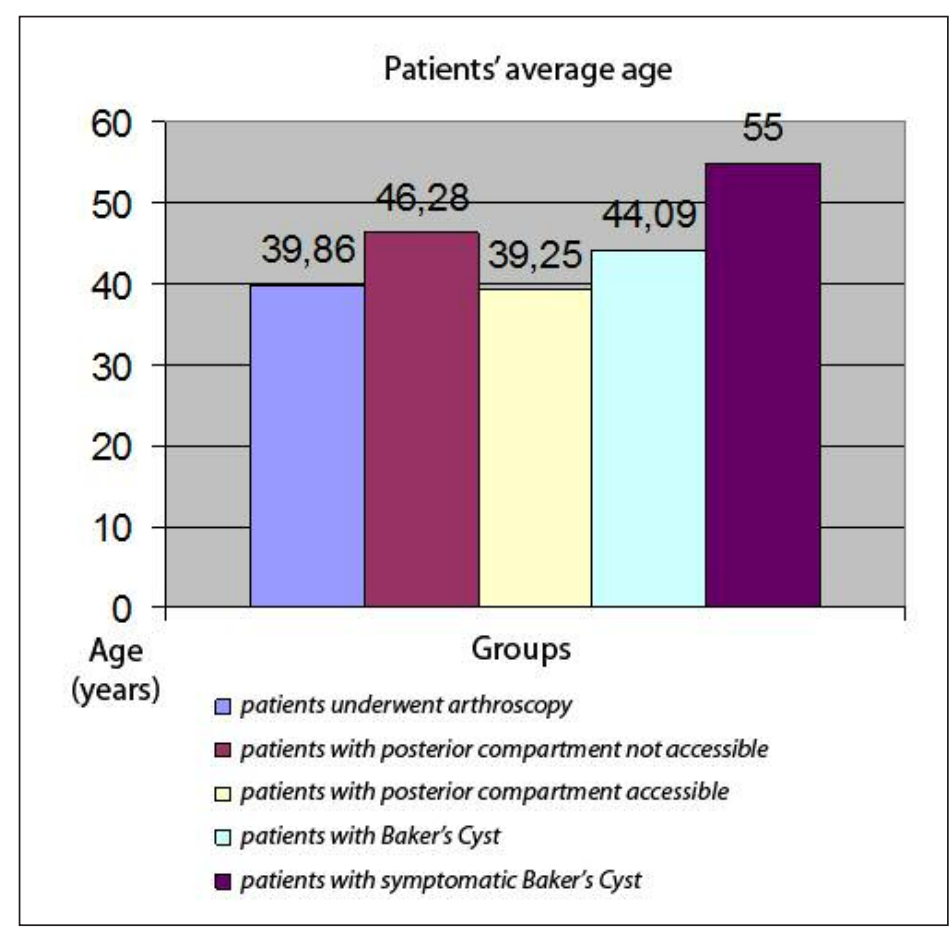

Figure 2. Average age distribution in the different subgroups.

A statistically consistent association between $\mathrm{BC}$ and chondropathy was present $(p=0.046)$. The relative risk associated with chondropathy was 1.94 .

\section{Discussion}

The GSB is situated between the tendons of the gastrocnemius and semimembranosus muscles. Some- times it can communicate with the joint with a transverse opening in the posterior capsule at the level of the medial condyle 8,9 .

The prevalence of a communication of the GSB with the knee joint in adults varies in relation to the type of study and the population: $30-50 \%$ in cadaveric dissections, $55 \%$ in open surgical excision, $37 \%$ in knee diagnostic arthroscopies and $50 \%$ in arthrograms of normal knees ${ }^{10,11}$. 


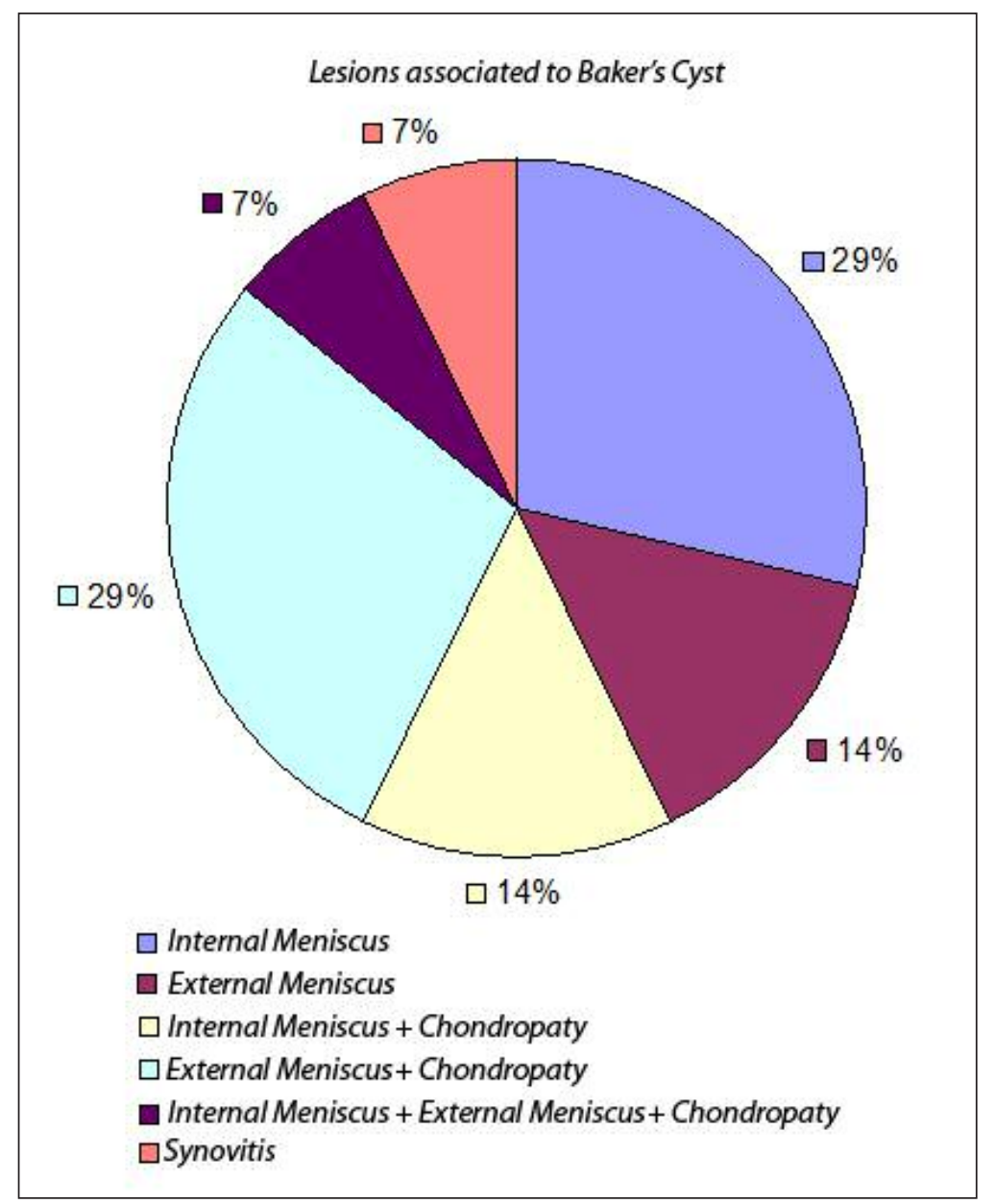

Figure 3. Lesions associated with the BC.

Johnson et al. report a prevalence of $37 \%$ in an adult asymptomatic population but they refer to only the popliteal bursa ${ }^{12}$; other studies report a prevalence of about $20 \%$ in symptomatic patients as well as correlation with intra-articular pathologies such as meniscopathy, sinovitis and osteochondrosis ${ }^{13-16}$. Lindgren and Willen reported a prevalence of $50 \%$ in adults ${ }^{8}$. This communication is actually unknown in children whereas its prevalence increases with age, so it is hypothesized that it results from a degenerative tear in the posterior capsule 8,17 .

Furthermore, even where the opening was not present, a thinning of the posterior capsule was noted ${ }^{18}$. When the communication exists, a chronic synovitis, sustained by intra-articular disorders, may cause recurrent effusions to fill the GSB with fluid that becomes trapped inside the GSB. The GSB progressively enlarges and forms a pseudocystic cavity. At this stage the term Baker's cyst should be preferred $^{1,4}$.

The presence of a valve mechanism has been demonstrated by several Authors, but the nature of the valve is the subject of some debate. Some Authors sustain that it is a Bunsen-type mechanism ${ }^{17}$. Other Authors hypothesize that the valve is created by the interaction of the medial gastrocnemius tendon, and the semimembranosus and semitendinosus tendons ${ }^{18}$

It has been reported that the intra-articular pressure in knee joints with effusions is around $33 \mathrm{~mm} \mathrm{Hg}$ at rest reaching peaks of up to $1,300 \mathrm{~mm} \mathrm{Hg}$ under given conditions ${ }^{10}$. When such high pressures occur, the opening of the gateway to the GSB during flexion may form a conduit through which synovial fluid is pumped into the bursa; conversely, the aperture closes during extension. If the fluid trapped and stagnating inside the GSB becomes denser, as is often the case in chronic Baker's cysts, the increased viscosity may hinder a retrograde outflow. In other words, the alleged unidirectional valve-like mechanism may, in some instances, be functional and not anatomical.

Epidemiological studies have shown that Baker's cysts are often associated with knee joint disorders ${ }^{1,3,12}$. This association was also observed in our study as well as in the case series published by Sansone and De Ponti ${ }^{19}$. In the presence of an anatomical connection the pathogenesis of a Baker's cyst to be advocated would be a one-way valve-like mechanism and the interplay of differential pressures between the GSB and the knee joint during kinematics ${ }^{10}$. 
A consistent correlation should be present with pathologies of the medial compartment; the internal derangement results from disturbed biomechanics with the increased pressure causing normal joint fluid to flow into the bursa ${ }^{20,21}$; otherwise, no correlation should be present with the knee effusion grade ${ }^{14}$.

In our study, the prevalence of BC was $28.3 \%$. Given such a percentage, the exploration of the posterior aspect of the knee has to be performed routinely, even though MRI is particularly sensitive in identifying its presence preoperatively ${ }^{19,22}$.

Unfortunately, it is not possible to perform this maneuver in all cases; a particularly narrow notch for a specific anatomy or, more frequently, the presence of osteophytes, can prevent the passage of the arthroscope between the medial condyle and the posterior cruciate ligament.

In fact, the average age of the group where posterior arthroscopic exploration was not possible is higher $(a=0.05)$, and this is probably due to the presence of osteophytes in this population.

For the purposes of the exploration, a $70^{\circ}$ lens permits better exploration and visualization of the cystic hiatus.

Indeed, a previous notchplasty could be helpful to permit the exploration of the posterior compartment, but sometimes this procedure might not suffice. Several alternative techniques and portals are proposed in the literature to provide for better access ${ }^{23}$.

Lysholm and Gillquist firstly proposed the technique for visualizing the posterior compartment via the intercondylar notch; nevertheless, they used a central transpatellar tendon approach ${ }^{24}$.

Tolin and Sapega reported that a posteromedial approach in conjunction with the anteromedial approach allowed a better overall visualization ${ }^{25}$.

A trans-septal portal was then developed to improve the visualization of the posterosuperior aspect of the femoral condyles, the posterior portion of the posterior cruciate ligament, the entire periphery of the posterior horn of the meniscus, the posterior meniscofemoral ligament and the posterior aspect of the capsule 26 .

The arthroscopic posterior aspect of the knee was first described by Johnson et al. in $1997^{12}$. They identified the presence of a BC in 37 out of 195 knees and classified it in three possible patterns based on the presence of a transverse band and an opening into a popliteal bursa. They classified a pattern where there was neither a capsular folder nor an opening as Type I, those with a capsular folder without opening as Type II, and patterns with a capsular folder and opening as Type III.

A recent paper of Kim et al. has confirmed a relationship between the type of anatomy in the posteromedial capsule and the popliteal cyst; the cyst is present in $60 \%$ of type II and $66.7 \%$ of type III knees, whereas it is present in just $1.9 \%$ of type I knees ${ }^{27}$.

The posterior view of the knee joint depends on the relationship that the joint capsule and the synovia have with the OPL and the medial gastrocnemius ten- don. The study being presented describes six possible patterns; in knee types $\mathrm{C}, \mathrm{D}$ and $\mathrm{F}, \mathrm{BC}$ was always present. Some aspects of type $E$ knees can simulate the presence of the cyst that is, actually, not present.

In such cases, the exploration and palpation of the OPL and the medial gastrocnemius tendon is very important in order to confirm whether the cyst is present, and to provide for its treatment.

The high anatomic variability underlines the importance of an exhaustive classification of the local anatomy, so that the arthroscopic surgeon can easily recognize the $\mathrm{BC}$ and proceed with its treatment ${ }^{6}$.

Differential diagnosis has to be done versus all pathologies which can determine a swelling in the popliteal fossa as pigmented villonodular synovitis $^{28,29}$, tumors ${ }^{30,31}$ and vascular diseases ${ }^{32}$. Clinical examination and MRI are often sufficient to do diagnosis but biopsy is mandatory in case of tumor's suspect $^{33}$.

The treatment of symptomatic BC can be conservative or surgical ${ }^{34}$. The conservative treatment is sustained by few Authors which report good success rate but those results are probably not accepted by the most of the Authors who prefer surgical approach; infiltrative therapy could be more effective when intrarticular disease is at an early stage ${ }^{35}$. Recently, Smith et al. published the results of ultrasound-guided aspiration, fenestration and injection of $48 \mathrm{BCs}$ at a median follow-up of 106.5 weeks; they reported a recurrence rate of $12.7 \%$ identifying advanced osteoarthritis as the main risk factor ${ }^{36}$.

Surgery may be required if symptoms related to the cyst persist and limit patients' knee function ${ }^{10}$.

In our opinion, surgery is indicated when BC becomes symptomatic because of compression of surrounding structures. Several surgical approaches are available; the most of the Authors sustains the importance of treating intrarticular disease which, probably, causes the onset of the cyst. Our group and other surgeons prefer also to close the gateway $6,38,39$, otherwise other Authors suggest to enlarge the communication, removing the valve mechanism ${ }^{40-43}$. A recent review analyzed 11 studies valuing the results of the surgical approach; it evidenced a success rate of 96.7 and $84.6 \%$ in the communication-enlargement group and communication-closure group, respectively; considering just the first group, the percentage of success was $98.2 \%$ for the Authors sustaining resection of the cyst wall and $94.7 \%$ for the Authors which don't do it ${ }^{44}$.

The arthroscopic approach assumes greater importance when we consider the high incidence of recurrence of $B C$ after its removal by the posterior approach and without treating the associated intra-articular pathologies ${ }^{18}$.

We believe that the first step in treating a symptomatic Baker's cyst is the performance of a meticulous diagnostic arthroscopy, and this should be followed by treatment of those disorders that provoke chronic synovitis. However, not all disorders may be defini- 
tively treated. For example, where diffuse chondral lesions and secondary hypertrophy of the synovial membrane occur in middle-aged patients, the knee is predisposed to recurrent effusions and the cyst may eventually recur.

\section{Conflicts of interest}

The Authors declare no conflicts of interest concerning this article.

\section{References}

1. Wilson PD, Eyre-Brook AL, Francis JD. A clinical and anatomical study of the semimembranosus bursa in relation to popliteal Cyst. J Bone Joint Surg. 1938;20:963-84.

2. Hayashi D, Roemer FW, Dhina Z, Kwoh CK, Hannon MJ, Moore C, Guermazi A. Longitudinal assessment of cyst-like lesions of the knee and their relation to radiographic osteoarthritis and MRI-detected effusion and synovitis in patients with knee pain. Arthritis Res Ther. 2010;12(5):R172.

3. Trecco F, De Paulis F, Masciocchi C, et al. Clinical interpretation of cysts in the popliteal space using computerised tomography. Ital J Orthop Traumatol. 1984;10:109-120.

4. Baker WM. On the formation of the synovial cysts in the leg in connection with disease of the knee joint. St Bartholomew's Hospital Report, London. 1877;13:245-261.

5. Herman AM, Marzo JM. Popliteal cysts: a current review. Orthopedics. 2014;37(8):e678-84.

6. Calvisi V, Lupparelli S, Giuliani P. Arthroscopic all-inside suture of symptomatic Baker's cysts: a technical option for surgical treatment in adults. Knee Surg Sports Traumatol Arthrosc. 2007;15(12):1452-1460.

7. Padulo J, Oliva F, Frizziero A, Maffulli N. Muscles, Ligaments and Tendons Journal - Basic principles and recommendations in clinical and field Science Research: 2016 Update. MLTJ. 2016;6(1):1-5.

8. Lindgren PG, Willén R. Gastrocnemiosemimembranosus bursa and its relation to the knee joint: I. Anatomy and histology. Acta Radiol Diagn (Stockh). 1977;18:497-512.

9. Rauschning W. Anatomy and function of the communication between knee joint and popliteal bursae. Ann Rheum Dis. 1980;39:354-358.

10. Handy JR. Popliteal cysts in adults: a review. Semin Arthritis Rheum. 2001;31(2):108-118.

11. Frush TJ, Noyes FR. Baker's Cyst: Diagnostic and Surgical Considerations. Sports Health. 2015;7(4):359-365.

12. Johnson LL, van Dyk GE, Johnson CA, Bays BS, Gully SM. The popliteal bursa (Baker's cyst): an arthroscopic perspective and the epidemiology. Arthroscopy. 1997;13:66-72.

13. Fielding JR, Franklin PD, Kustan J. Popliteal cysts: a reassessment using magnetic resonance imaging. Skeletal Radiol. 1991;20(6):433-435.

14. Gristina AG, Wilson PD. Popliteal cysts in adults and children. A review of 90 cases. Arch Surg. 1964;88:357-363.

15. Nicholas JA, Freiberger RH, Killoran PJ. Double-contrast arthrography of the knee. Its value in the management of two hundred and twenty-five knee derangements. J Bone Joint Surg Am. 1970;52(2):203-220.

16. Bacon PA, Gerber NJ. Popliteal cysts and synovial rupture in osteoarthrosis. Rheumatol Rehabil. 1974;13(2):98-100.

17. Jayson MI, Dixon AS. Valvular mechanisms in juxta-articular cysts. Ann Rheum Dis. 1970;29:415-420.

18. Rauschning W, Lindgren PG. Popliteal cysts (Baker's cysts) in adults. I. Clinical and roentgenological results of operative ex- cision. Acta Orthop Scand. 1979;50(5):583-591.

19. Sansone V, de Ponti A, Paluello GM, del Maschio A. Popliteal cysts and associated disorders of the knee. Critical review with MR imaging. Int Orthop. 1995;19(5):275-279.

20. Miller TT, Staron RB, Koenigsberg T, Levin TL, Feldman F. MR imaging of Baker cysts: association with internal derangement, effusion and degenerative arthropathy. Radiology. 1996;201:247-450.

21. Vasilevska V, Szeimies U, Staebler A. MRI diagnosis of Baker cyst and significance of associated medial compartment knee osteoarthritis. Radiol Oncol. 2008;42(2):51-58.

22. Trieshmann HW Jr, Mosure JC. The impact of magnetic resonance imaging of the knee on surgical decision making. Arthroscopy. 1996;12(5):550-555.

23. Landsiedl F, Aigner N, Hexel M, Krasny C, Schenk S, Steinböck $\mathrm{G}$. A new arthroscopic technique for revision of the posterior compartment in symptomatic total knee arthroplasty. Arthroscopy. 2005;21(4):506-510.

24. Lysholm J, Gillquist J. Arthroscopic examination of the posterior cruciate ligament. J Bone Joint Surg Am. 1981;63:363-366.

25. Tolin BS, Sapega AA. Arthroscopic visual field mapping at the periphery of the medial meniscus: a comparison of different portal approaches. Arthroscopy. 1993;9:265-271.

26. Ahn JH, Ha CW. Posterior trans-septal portal for arthroscopic surgery of the knee joint. Arthroscopy. 2000;16:774-779.

27. Kim KI, Lee SH, Ahn JH, Kim JS. Arthroscopic anatomic study of posteromedial joint capsule in knee joint associated with popliteal cyst. Arch Orthop Trauma Surg. 2014;134(7):979984.

28. Oliva F, Frizziero A. One step open synovectomy without adjuvant therapy for diffuse pigmented villonodular synovitis of the knee in a soccer player. Muscles Ligaments Tendons $\mathrm{J}$. 2011;1(1):36-39.

29. Tosti R, Kelly JD 4th. Pigmented villonodular synovitis presenting as a baker cyst. Am J Orthop (Belle Mead NJ). 2011;40(10):528-531.

30. Komurcu E, Golge UH, Kaymaz B, Erdogan N. Popliteal Schwannoma mimicking baker cyst: an unusual case. J Surg Case Rep. 2013;2013(8).

31. Choi YJ, Kang JH, Kang GH, Choi SJ. Osteochondrolipoma Presenting as a Popliteal Cyst. Clin Orthop Surg. 2015;7(2): 264-8.

32. Allemang MT, Kashyap VS. Adventitial cystic disease of the popliteal artery. J Vasc Surg. 2015;62(2):490.

33. Perdikakis E, Skiadas V. MRI characteristics of cysts and "cyst-like" lesions in and around the knee: what the radiologist needs to know. Insights Imaging. 2013;4(3):257-272.

34. Herman AM, Marzo JM. Popliteal cysts: a current review. Orthopedics. 2014;37(8):e678-684.

35. Bandinelli F, Fedi R, Generini S, et al. Longitudinal ultrasound and clinical follow-up of Baker's cysts injection with steroids in knee osteoarthritis. Clin Rheumatol. 2012;31(4):727-731.

36. Smith MK, Lesniak B, Baraga MG, Kaplan L, Jose J. Treatment of Popliteal (Baker) Cysts With Ultrasound-Guided Aspiration, Fenestration, and Injection: Long-term Follow-up. Sports Health. 2015;7(5):409-414.

37. Rauschning W. Popliteal cysts (Baker's cysts) in adults. II. Capsuloplasty with and without a pedicle graft. Acta Orthop Scand. 1980;51(3):547-555.

38. Hughston JC, Baker CL, Mello W. Popliteal cyst: a surgical approach. Orthopedics. 1991;14(2):147-50.

39. Ko S, Ahn J. Popliteal cystoscopic excisional debridement and removal of capsular fold of valvular mechanism of large recurrent popliteal cyst. Arthroscopy. 2004;20(1):37-44.

40. Cho JH. Clinical results of direct arthroscopic excision of popliteal cyst using a posteromedial portal. Knee Surg Relat Res. 2012;24(4):235-240.

41. Ahn JH, Lee SH, Yoo JC, Chang MJ, Park YS. Arthroscopic 
treatment of popliteal cysts: clinical and magnetic resonance imaging results. Arthroscopy. 2010;26(10):1340-1347.

42. Ohishi T, Takahashi M, Suzuki D, et al. Treatment of popliteal cysts via arthroscopic enlargement of unidirectional valvular slits. Mod Rheumatol. 2015;25(5):772-778.

43. Kongmalai $\mathrm{P}$, Chernchujit B. Arthroscopic Treatment of
Popliteal Cyst: A Direct Posterior Portal by Inside-Out Technique for Intracystic Debridement. Arthrosc Tech. 2015; 4(2):e143-148.

44. Zhou XN, Li B, Wang JS, Bai LH. Surgical treatment of popliteal cyst: a systematic review and meta-analysis. J Orthop Surg Res. 2016;11:22. 